\title{
Dental Caries Occurrence in Inflammatory Bowel Disease Patients: A Systematic Review and Meta-Analysis
}

\author{
Crystal Marruganti ${ }^{a}$ b Nicola Discepoli ${ }^{b}$ Carlo Gaeta $^{a}$ Giovanni Franciosi ${ }^{a}$ \\ Marco Ferrari ${ }^{c}$ Simone Grandini ${ }^{a}$ \\ aDepartment of Medical Biotechnologies, Unit of Endodontics and Restorative Dentistry, University of Siena, Siena, \\ Italy; ${ }^{b}$ Department of Medical Biotechnologies, Unit of Periodontology, University of Siena, Siena, Italy; ' ${ }^{\text {Department }}$ \\ Medical Biotechnologies, Unit of Prosthodontics, University of Siena, Siena, Italy
}

\author{
Keywords \\ Dental caries · Inflammatory bowel disease $\cdot$ Systematic \\ review
}

\begin{abstract}
Objective: The present review aimed to systematically evaluate the occurrence of caries in patients with inflammatory bowel disease (IBD), either Crohn's disease (CD) or ulcerative colitis (UC), compared to healthy controls. Materials and Methods: MEDLINE (PubMed), Embase, Google Scholar, LILACS, and Cochrane Library electronic databases were screened. Caries experience was measured through the Decayed, Missing, Filled Teeth (DMFT) index. The weighted mean difference (WMD) with 95\% confidence interval was calculated between IBD patients and healthy controls. $R \boldsymbol{e}$ sults: Six studies were selected for the inclusion in the systematic review, 5 of which were also included in the quantitative synthesis of data. The WMD in the DMFT index between IBD and healthy subjects was $3.04(1.52,4.56)(p=$ $0.10)$. Subgroup analysis showed no difference $(p=0.31)$ between CD $(2.52[0.54,4.49])$ and UC $(4.01[1.52,4.56])$ subjects. Conclusions: There is a remarkably higher past and present occurrence of dental caries in subjects with IBD than
\end{abstract}

healthy controls. This result should encourage clinicians to include oral health preventive programs in the overall treatment plan of IBD patients.

(c) 2021 S. Karger AG, Basel

\section{Introduction}

Within the definition of inflammatory bowel diseases (IBD), 2 chronic, relapsing inflammatory processes of the gastrointestinal tract are encompassed: Crohn's disease (CD) and ulcerative colitis (UC). While inflammation in UC is characteristically described to involve both the colonic mucosal surface [Ordás et al., 2012] and the oral mucosa (in the form of aphthous stomatitis) [Dotson et al., 2010; Ordás et al., 2012] in CD, it retains a nonuniform transmural extension along any part of the gastrointestinal tract [Baumgart and Sandborn, 2012]. The current paradigm of IBD pathogenesis comprises a combination of aberrant immune response, genetic susceptibility, gut dysbiosis, and environmental factors such as smoking, antibiotics, oral contraceptives, and diet [Abraham and Cho, 2009; Chapman-Kiddell et al., 2010]. The Westernization of lifestyle and diet, with considerably higher 
intake of refined sugars, was appointed as a key factor in the escalating incidence of IBD across Europe and the rest of the world [Ooi et al., 2016].

The most frequent symptoms of IBD include abdominal pain, diarrhea, and rectal bleeding [Shivashankar and Lichtenstein, 2018]. Between $6 \%$ and $47 \%$ of patients were also reported to develop extra-intestinal manifestations of IBD, $25 \%$ of which occurred a median time of 5 months prior to IBD diagnosis and 75\% occurred afterward [Vavricka et al., 2015]. Extra-intestinal manifestations most frequently include arthropathies, skin diseases, cholangitis, and oral lesions such as aphthous ulcers and periodontitis. Indeed, a recent meta-analysis showed that the odds of periodontitis in IBD patients are 3-fold greater than those in IBD-free controls [She et al., 2020]. Some studies also reported an increased prevalence of dental caries in patients with IBD [Rooney, 1984; Brito et al., 2008; Ślebioda et al., 2011; Vavricka et al., 2013; Szymanska et al., 2014].

Many hypotheses were proposed to explain the biological plausibility of the link between IBD and caries. First of all, some studies reported an increased salivary composition of Streptococcus mutans and Lactobacilli spp. in IBD patients compared to IBD-free controls [Szymanska et al., 2014; Rodrigues et al., 2019]. This observation may be related to either the dietary changes during the active phases of IBD disease (i.e., increased sugar consumption) [Järnerot et al., 1983] and the gut dysbiosis with reduced microbial diversity typical of both CD and UC. Nonetheless, the association between caries and IBD has not been clearly elucidated yet. Confirmation of such association would pave the way for the promotion of activities targeted at the early detection and prevention of dental caries since the moment of IBD diagnosis. Moreover, the creation of behavioral interventions targeted to the common risk factors control [Sheiham and Watt, 2000] of IBD and dental caries could implement the long-term management of both diseases. Therefore, the current review aimed to systematically evaluate the occurrence of caries in patients with IBD compared to IBD-free controls.

\section{Methods}

\section{Protocol and Registration}

The following systematic review was performed according to the PRISMA statement [Page et al., 2021] and received the registration number from the Prospective Register of Systematic Reviews (CRD42020213441).

\section{Eligibility Criteria}

Studies investigating the presence of caries in patients with either CD or UC were included in the present review. The Popula- tion, Exposure, Control, Outcome, and Studies method was used to identify the eligibility criteria:

The exclusion criteria of studies were as follows: reviews or register study designs, lack of control group (healthy subjects), outcome measure (DMFT), or disease definition (either CD or UC).

\section{Information Sources}

The search strategy included the screening of electronic databases and hand searching in relevant journals and other sources, for example, references in reviews related to our research question. The screening and inclusion steps were reported according to the PRISMA flow diagram [Page et al., 2021].

\section{Electronic Search}

MEDLINE, Embase, Google Scholar, LILACS, and Cochrane Central Register of Controlled Trials electronic databases were screened. A search in grey literature (OpenGrey) was also carried out. The search strategy was built with a combination of $\mathrm{MeSH}$ terms and free-text words. Only articles in English were considered for the inclusion in the study, while no publication date restrictions were applied during the search. The full electronic strategy applied is reported for MEDLINE (through PubMed):

The search strategy was then modified for the other databases.

\section{Hand Search}

Hand search was performed independently by 2 calibrated investigators (C.M. and C.G.) in relevant journals (International Endodontic Journal, Journal of Endodontics, Journal of Clinical Periodontology, Journal of Periodontology, and Inflammatory Bowel Diseases) evaluating issues published between January 1980 and April 2021. Other sources encompass narrative reviews and references of relevant articles.

\section{Study Selection}

Articles were first screened by title and abstract by 2 independent and calibrated reviewers (C.M. and C.G.) (unweighted Cohen's kappa score of 0.90). Whenever information was not clear at this stage, articles were selected for full-text analysis and reviewed by both investigators according to the inclusion and exclusion criteria. Any disagreement at this stage was resolved through discussion with a third author (S.G.).

\section{Data Collection Process}

Data collection was carried out through an extraction sheet by 2 independent reviewers (C.M. and C.G.) during full-text analysis. Study characteristics included: country, study design, matched variables, number of participants (proportions of males and females), age, disease definition, disease activity (IBD), and dietary habits (meal frequency). Authors were contacted whenever information was unclear at this stage.

\section{Data Items}

The primary outcomes of the present review protocol were the presence of caries in patients with IBD versus healthy subjects. The presence of caries was evaluated through the DMFT index [World Health Organization, 2013]. Weighted mean differences (WMDs) in DMFT values between IBD and control subjects were then computed. 


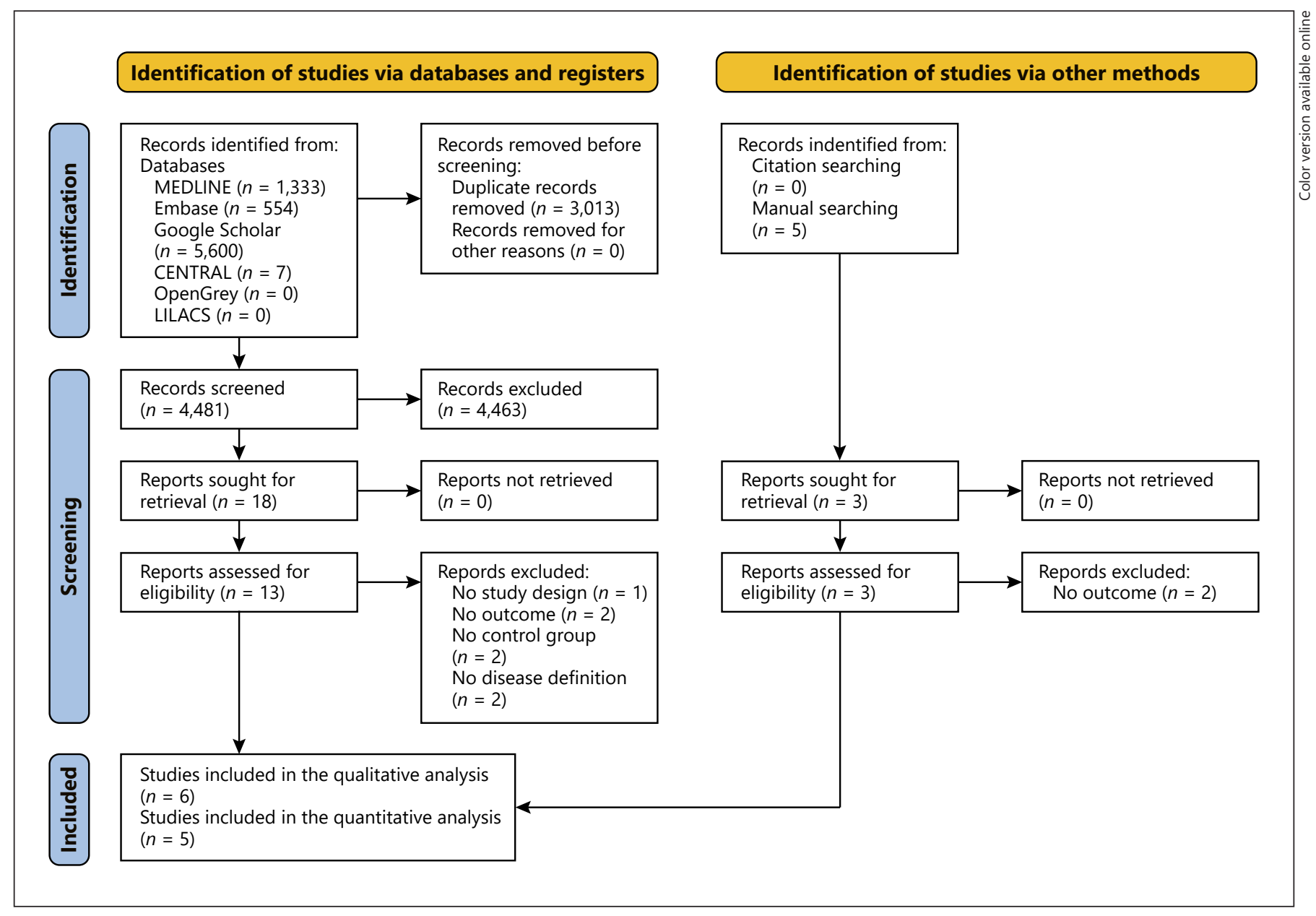

Fig. 1. PRISMA flow diagram summarizing all inclusion stages.

\section{Risk of Bias in Individual Studies}

Overall quality of the included studies was assessed with the Newcastle-Ottawa Scale (NOS) for case-control studies [Wells et al., 2009].

It encompasses 3 categories:

1. selection of cases and controls (4 items);

2. comparability of cases and controls on the basis of the design or analysis (1 item);

3. exposure (3 items).

Each study was given a maximum of one star per each item of the Selection and Exposure categories and a maximum of 2 stars in the Comparability section. The overall quality for each study was therefore assessed on a scale of 0-9 points. Whenever a summary score $\geq 5$ was reached, the study was rated as moderate or high quality. Cross-sectional studies were evaluated through the Critical Appraisal Checklist for Analytical Cross-sectional studies (Joanna Briggs Institute, University of Adelaide; http://joannabriggs.org/research/critical-appraisal-tools.html); it consists of 8 questions with 4 possible answers: yes ("low risk of bias"), no ("high risk of bias"), unclear ("unclear risk of bias"), and not applicable. Any disagreement between investigators at this stage was resolved through discussion; in case consensus was not reached, a third investigator (S.G.) was involved. Inter-examiners agreement was calculated using the kappa score.

\section{Summary Measures}

Data were pooled for both qualitative and quantitative analysis. The DMFT index was considered as the primary outcome measure and reported as mean and standard deviation. Whenever it was not present, an attempt was made to contact authors and obtain raw data. The estimate of the effect was calculated as the WMD for the DMFT index.

\section{Risk of Bias across Studies}

The overall quality of evidence at the outcome level (DMFT index) was evaluated using the Grades of Recommendation, Assessment, Development, and Evaluation approach [Guyatt et al., 2011]. The quality of evidence was rated on a four-level scale (very low, low, moderate, and high) according to study design, risk of bias, inconsistency, indirectness, and imprecision; each of these items was given a judgment (very serious, serious, and not serious) [Guyatt et al., 2009a]. On these grounds, the strength of recommendation was deemed critical, important, or not important [Guyatt et al., 2009b]. 


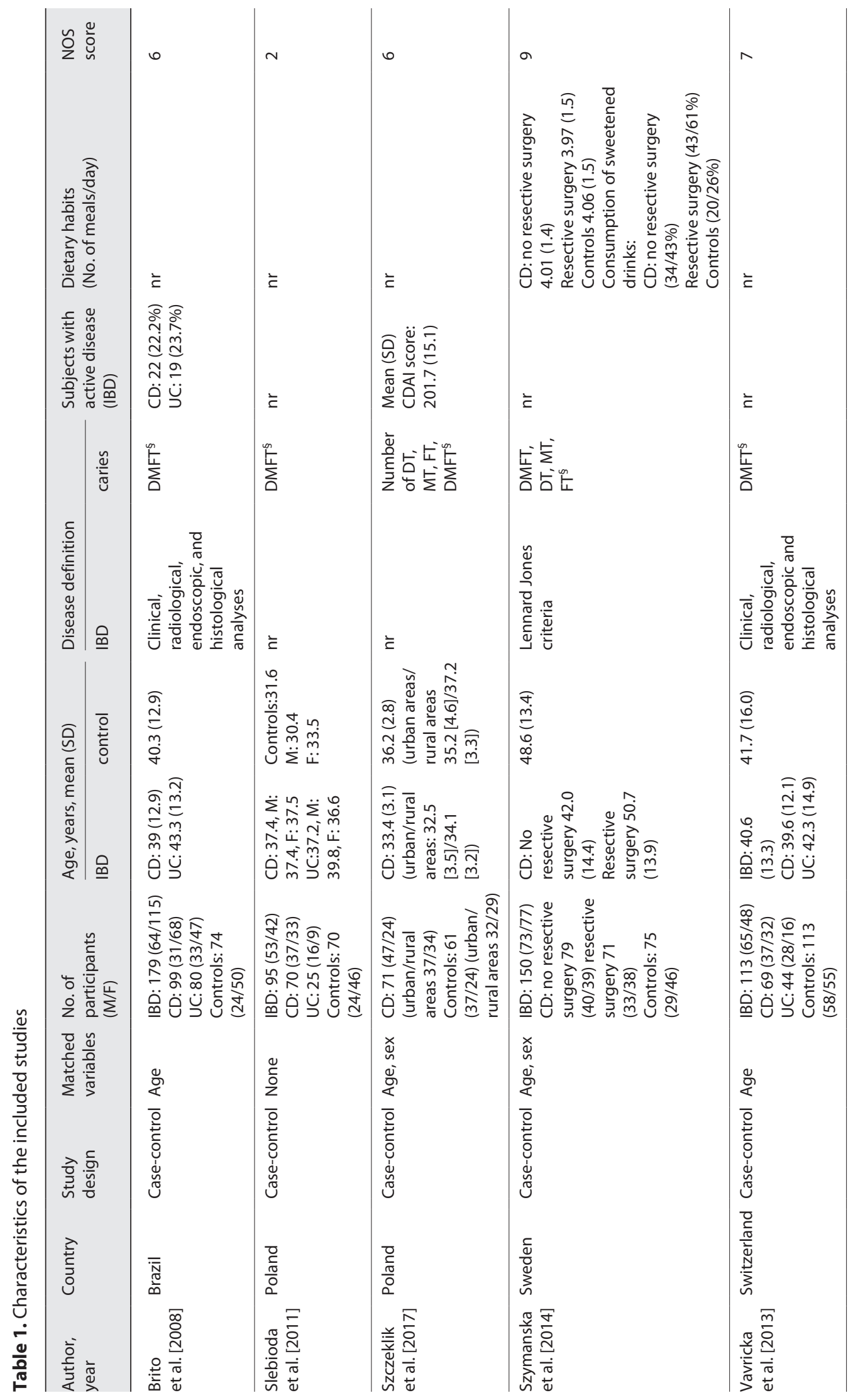




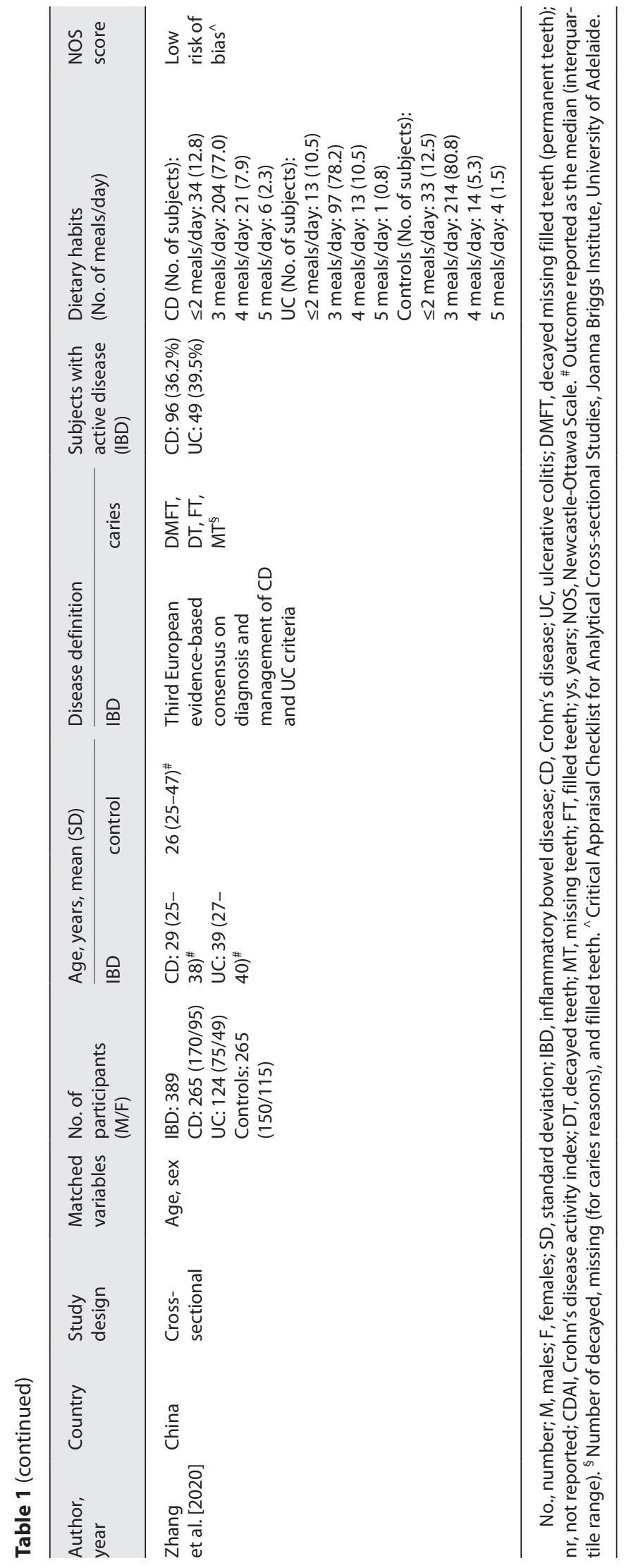

Synthesis of Results and Additional Analyses

All analyses were performed using an ad hoc statistical software (version 16.1, STATA IC, Stata Corp) setting the level of significance at $\alpha=0.05$. The $Q$ test based on $\chi^{2}$ statistics as well as the $I^{2}$ index were used in order to identify the percentage of variation in the global estimate attributable to heterogeneity. It was defined as: absent $\left(I^{2}: 0-25 \%\right)$, low $\left(I^{2}: 25.1-50 \%\right)$, moderate $\left(I^{2}: 50.1-75 \%\right)$, or high $\left(I^{2}: 75.1-100 \%\right)$. Due to the high heterogeneity across studies, the DerSimonian and Laird random-effect model [DerSimonian and Laird, 1986] was applied to conduct the meta-analysis using the means and standard deviations for DMFT values.

The DMFT index for each study was expressed as the WMD with $95 \%$ confidence interval (95\% CI) between cases and controls, and then, a subgroup analysis was performed according to disease definition (CD or UC). Publication bias was assessed through the Egger's test and visualized as funnel plot. Additionally, the jackknife sensitivity analysis omitted one study at a time and investigated whether one study had an exaggerated effect on the pooled estimates.

\section{Results}

\section{Study Selection}

Initial electronic search retrieved 1,333 records in MEDLINE, 554 in Embase, 5,600 in Google Scholar, 7 in Cochrane Central Register of Controlled Trials, and 0 in OpenGrey and LILACS. Hand-search from relevant journals led to 5 additional articles and none from other sources. After duplicates removal, 18 records were selected at the abstract level according to the inclusion and exclusion criteria. Thirteen full-text articles were assessed for eligibility, 6 of which were included in the qualitative analysis. All studies except for one [Zhang et al., 2020] were included in the quantitative (meta-analysis) synthesis of data. Reasons and timing of exclusion for each study are presented in online supplementary File 1 (see www. karger.com/doi/10.1159/000519170 for all online suppl. material). Investigators agreement for articles inclusion resulted in a $\mathrm{k}$ score of 0.90 . A PRISMA flow diagram summarizing all inclusion stages is presented in Figure 1.

\section{Study Characteristics}

Characteristics of the included studies [Brito et al., 2008; Ślebioda et al., 2011; Vavricka et al., 2013; Szymanska et al., 2014; Szczeklik et al., 2017; Zhang et al., 2020] are shown in Table 1. Two studies were conducted in Poland [Ślebioda et al., 2011; Szczeklik et al., 2017], and the other 4 studies were conducted in Brazil, Sweden, Switzerland, and China, respectively, [Brito et al., 2008; Vavricka et al., 2013; Szymanska et al., 2014; Zhang et al., 2020]. One study [Zhang et al., 2020] had a cross-sectional design, while the others were case-control studies [Brito et al., 2008; Ślebioda et al., 2011; 


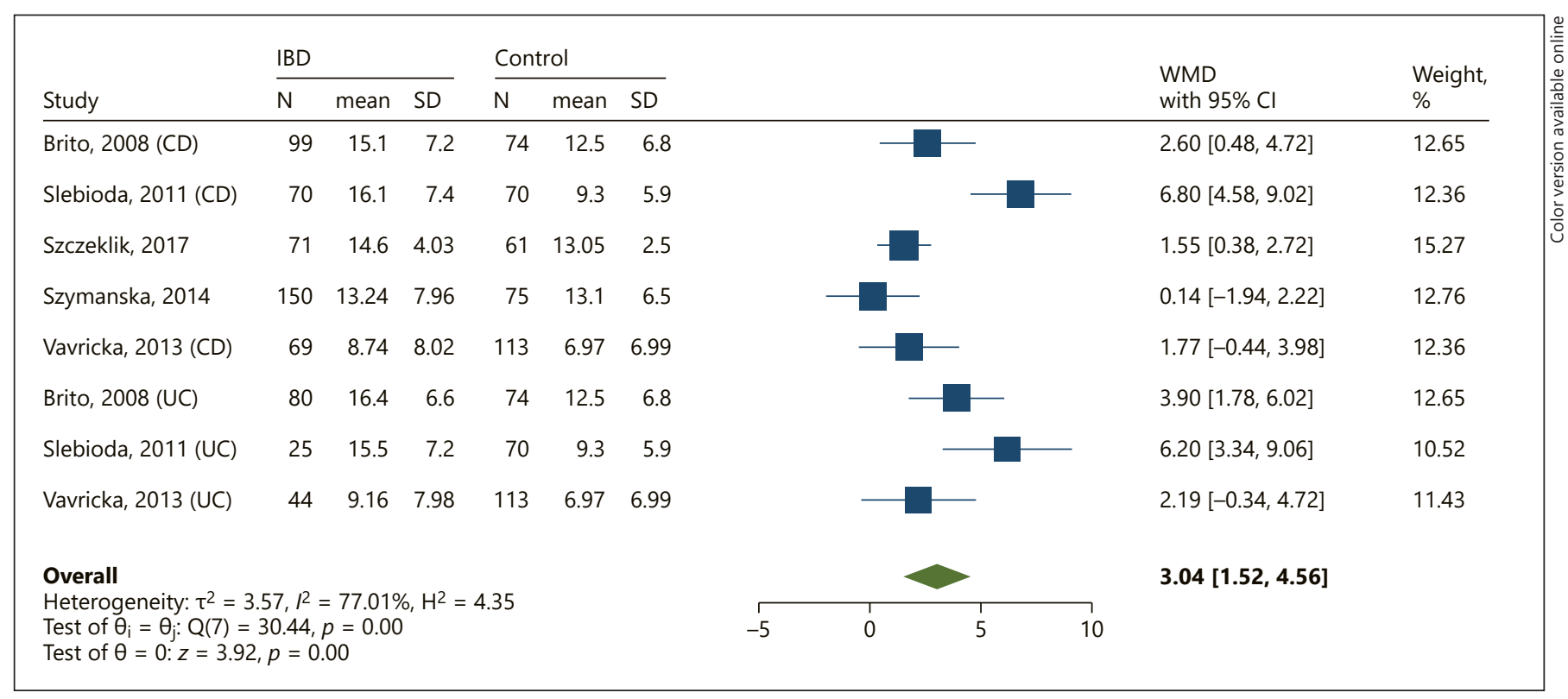

Fig. 2. DMFT levels (WMD 95\% CI) in IBD versus healthy controls (Random-effects DerSimonian-Laird model). DMFT, Decayed, Missing, Filled Teeth; WMD, weighted mean difference; CI, confidence interval; IBD, inflammatory bowel disease.

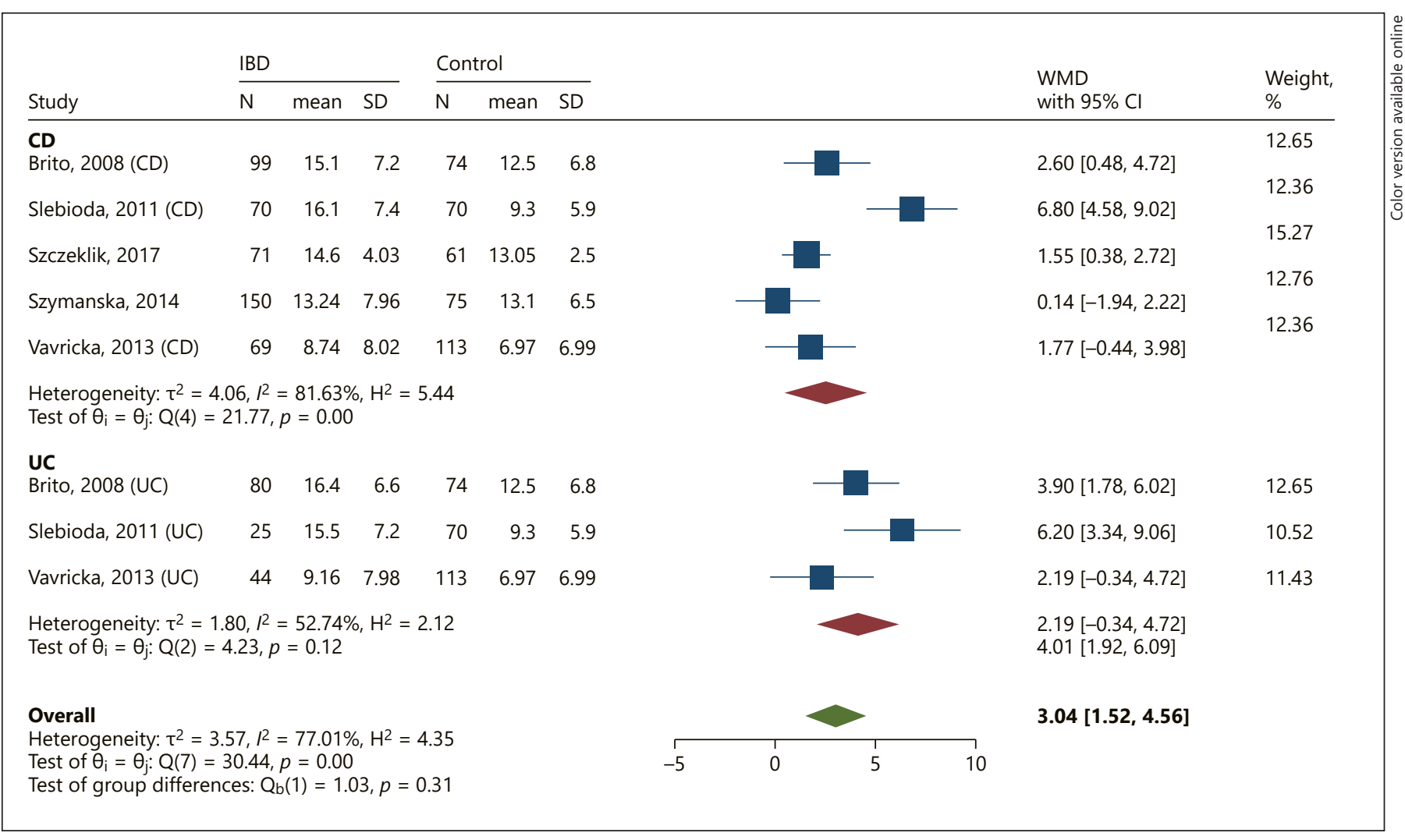

Fig. 3. Subgroup analysis according to disease definition (CD vs. UC) (Random-effects DerSimonian-Laird model). CD, Crohn's disease; UC, ulcerative colitis; WMD, weighted mean difference; CI, confidence interval; IBD, inflammatory bowel disease. 


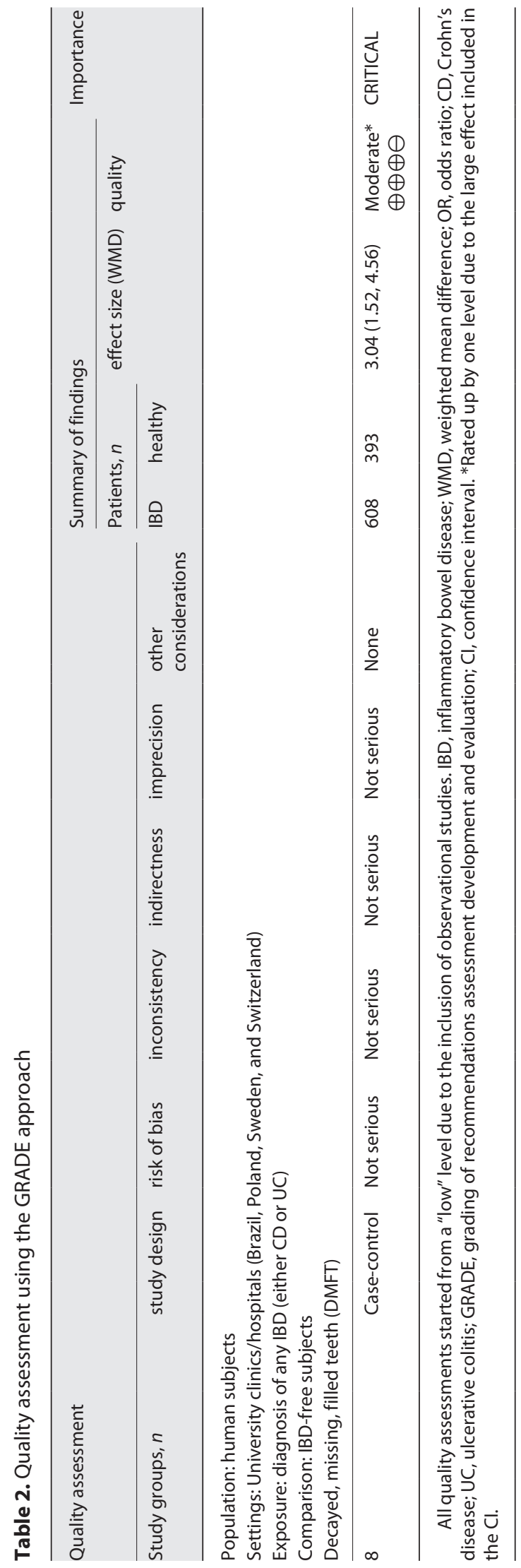

Caries and Inflammatory Bowel Disease
Vavricka et al., 2013; Szymanska et al., 2014; Szczeklik et al., 2017]. For the primary outcome (DMFT index), participants were a total of 997 IBD (age range: 29-46.1, proportion of males/females: 547/450) and 658 healthy subjects (age range: 26-48.6, proportion of males/females: 322/336).

\section{Risk of Bias in Individual Studies}

Inter-examiners agreement resulted in an unweighted Cohen's kappa score of 0.90 . Individual and summary scores according to the NOS for each study are presented in online supplementary File 2. All studies, except for one [Ślebioda et al., 2011], resulted in a moderate to high methodological quality (summary score $\geq 5$ ). In the Selection category, 4 out of 5 studies lacked the definition of controls (healthy subjects). On the other hand in the Comparability category, all studies except for one [Ślebioda et al., 2011] matched cases and controls for at least the most important factor (age); 3 studies out of 5 did not match patients for any additional factor (e.g., sex and socioeconomic status)[Brito et al., 2008; Ślebioda et al., 2011; Vavricka et al., 2013]. In the Exposure category, 3 studies out of 5 had different nonresponse rates for the 2 groups [Brito et al., 2008; Ślebioda et al., 2011; Szczeklik et al., 2017]. The risk of bias assessment of the cross-sectional study [Zhang et al., 2020] resulted in "low risk of bias" for all 8 items (online suppl. File 3).

\section{Results of Individual Studies and Synthesis of Data}

One study [Zhang et al., 2020] was excluded from the quantitative synthesis of data as the outcome measure could not be computed through raw data analysis with the available information. When comparing IBD to healthy controls, the WMD $(95 \% \mathrm{CI})$ in the DMFT index was 3.04 $(1.52,4.56)(p=0.00)$ (shown in Fig. 2). Subgroup metaanalysis according to disease definition (CD vs. UC) resulted in a WMD $(95 \% \mathrm{CI})$ of $2.52(0.54,4.49)$ for $\mathrm{CD}$ and of $4.01(1.92,6.09)$ for UC (shown in Fig. 3). No statistically significant differences were found between the 2 subgroups $(p=0.31)$. No publication bias was detected $(p=0.19)$; visual inspection of the funnel plot confirmed the presence of a symmetrical distribution of studies (online suppl. File 4). Results of the jack-knife sensitivity analysis (online suppl. File 5) did not significantly influence effect estimates, which ranged between 1.16 and 5.10 and remained statistically significant.

\section{Risk of Bias across Studies}

Results of the Grades of Recommendation, Assessment, Development, and Evaluation are presented in Table 2. All quality assessments started from a "low" level due to the 
inclusion of observational studies [Guyatt et al., 2009a]. The quality rating was rated up by one level due to the large effect included in the CI [Guyatt et al., 2009b]. Strength of recommendation was rated as "critical" for the DMFT index.

\section{Discussion/Conclusion}

\section{Summary of Evidence}

The current systematic review focuses on the population-based index of caries experience (DMFT) in patients with IBD compared to healthy controls. Overall, results showed significantly higher values of DMFT index in IBD patients versus healthy controls $(p<0.05)$; subgroup meta-analysis demonstrated no differences in the oral health status of CD and UC patients.

The increased caries experience in CD and UC patients compared to systemically healthy subjects could be attributed to various reasons. First of all, increased meal frequency as well as higher sugar and carbohydrates intakes were reported for both CD and UC patients [Järnerot et al., 1983; Schütz et al., 2003; Grössner-Schreiber et al., 2006; Szymanska et al., 2014; Głąbska et al., 2019]. In particular, patients during the active phases of $\mathrm{CD}$ tend to eat sugary foods more frequently as they plausibly ease the gastrointestinal symptoms associated with disease exacerbation [Järnerot et al., 1983]. Significantly higher daily sugar intakes were recently demonstrated also for patients with UC in remission versus non-IBD controls (mean: $20 \mathrm{~g} /$ day and $10.6 \mathrm{~g} /$ day, respectively) [Głąbska et al., 2019]. Only 2 of the included studies reported dietary habits registered through a questionnaire [Szymanska et al., 2014; Zhang et al., 2020]. Both studies did not highlight significant differences as to meal frequency; nonetheless, Szymanska and coworkers [Szymanska et al., 2014] demonstrated an increased consumption of sweetened drinks between meals in CD compared to non-IBD patients. Sugar consumption and meal frequency are undoubtedly related to caries risk [Sheiham and James, 2015]; indeed, they retain a huge variability across different countries and regions. The highest DMFT values in the present systematic review were registered in studies conducted in Eastern Europe [Ślebioda et al., 2011; Szczeklik et al., 2017]. It can be hypothesized that sugar intake played a pivotal role in this observation. In fact, the findings of a recent population-based cohort study from the European Crohn's and Colitis Organization (ECCO) Epidemiological Committee (EpiCom), demonstrated a significantly higher sugar intake in IBD patients from
Eastern Europe than in those from Western Europe [Burisch et al., 2014]. Nonetheless, sugar consumption was not registered in neither of the 2 studies conducted in Eastern Europe [Ślebioda et al., 2011; Szczeklik et al., 2017]. Additionally, changes in the salivary composition were also found to play a key role in the higher caries experience detected in IBD patients [Szymanska et al., 2014; Rodrigues et al., 2019]. Only one of the included studies analyzed the salivary microbiological composition in CD versus healthy subjects [Szymanska et al., 2014], thus demonstrating higher concentrations of Streptococcus mutans and Lactobacilli spp. in the saliva of CD patients versus controls. This is consistent with previous reports stating that while on one hand no variations of salivary flow rate and buffering capacity are present [Sundh and Emilson, 1989], on the other hand modifications in the concentration of acidogenic bacteria take place in both CD [Sundh and Emilson, 1989] and UC [Rodrigues et al., 2019] patients. However, evidence with this regard is still conflicting [Halme et al., 1993; Meurman et al., 1994]. Szymanska and coworkers [Szymanska et al., 2014] also highlighted a significant difference in caries experience, sweetened drinks consumption, and salivary counts of cariogenic bacteria between CD patients undergoing resective surgery and those who did not; hence, a relationship between the severity of the disease (leading to the need for surgery), a higher sugar intake, and thus an increased caries experience is suggested.

Part of the observed heterogeneity could also have been related to disease activity; as previously stated, IBD patients were reported to increase sugar consumption especially during the active phases of the disease [Järnerot et al., 1983]. Only 2 of the included studies recorded this information; in particular, Brito and coworkers [Brito et al., 2008] reported that almost half of the cases had active disease; moreover, Szczeklik and coworkers [Szczeklik et al., 2017] recorded a mean CD Activity Index score which is consistent with mildly active IBD [Jørgensen et al., 2005]. Indeed, DMFT values reported by both studies were among the highest for both CD and UC; therefore, a further relationship between disease activity and caries occurrence together with the mediating role of sugar intake can be hypothesized.

The present systematic review highlighted a burdensome past and present caries experience as well as an urgent need for dental treatments in patients with IBD; these findings should encourage health-care providers and policy makers to create oral health-care programs targeted at a high-risk stratum of society like subjects diagnosed with IBD. In particular, these programs should 
entail behavioral interventions for risk factors control (oral hygiene habits, sugar consumption, and meal frequency), as well as a prompt intervention to prevent the onset of the disease (primary prevention) or to treat the disease while at the early stages (secondary prevention) in order to avoid its clinical sequelae (e.g., apical periodontitis and tooth loss).

\section{Level of Evidence}

The body of evidence in individual studies was of moderate to high quality overall. One study only resulted in low quality [Ślebioda et al., 2011], while all the other studies achieved a NOS summary score consistent with moderate or high methodological quality. The most frequently violated item was the definition of controls; in fact, many of the included studies lacked an accurate description of the comparison group, thus leaving to question whether control subjects were exposed to the same risk factors and confounders as the cases and whether they were representative of the population that constituted the cases. Nonetheless, the efficiency of the adjustment for confounders was increased by matching [Stang, 2010]; indeed, all studies except for one [Ślebioda et al., 2011] matched for at least the most important factor (age). On the other hand, around half of the included studies had different numbers of IBD and non-IBD respondents; the different nonresponse rate was addressed by none of the studies, except for one [Szymanska et al., 2014], hence posing the risk for an overestimation of the effect of the exposure on the outcome.

Despite all ratings started from "low level" because of the observational nature of the included studies [Guyatt et al., 2009a], the overall assessment resulted in "moderate quality" due to the large effect included in the CI [Guyatt et al., 2009b]. All in all, the strength of recommendation as to the association between dental caries and IBD was judged as "critical." Therefore, these results should serve as a call to action for clinicians to guide IBD patients into structured oral health prevention and intervention programs from the moment of IBD diagnosis.

\section{Strengths and Limitations}

The strengths of the present review encompass the strict methodology used during every step according to the most recent guidelines [Page et al., 2021] as well as the fairly high number of included subjects (total of 1,655 subjects). Moreover, these findings constitute the first metadata so far regarding caries experience in $C D$ and UC patients separately. However, it presents some inherent limitations. First of all, a limited number of studies was included and therefore heterogeneity, whenever present, could not be fully addressed. In fact, a high amount of heterogeneity may be due to some inherent characteristics of the outcome variable (DMFT). Indeed, DMFT is a continuous-discrete variable ranging between 0 and 28; its values tend to be skewed to the right (positively skewed) and with an excess of zeros (with a possible thicker tail); as such, DMFT is seldom normally distributed. Moreover, part of the heterogeneity could be pointed out to the operator-dependency of the outcome measurement (DMFT) [World Health Organization, 2013] as well as other genetic and environmental factors influencing caries pathogenesis [Selwitz et al., 2007] that could not be accounted for in the meta-analysis. Furthermore, despite the pivotal role of diet [Sheiham and James 2015] and salivary characteristics (i.e., salivary flow rate, buffering capacity, and count of cariogenic bacteria) [Gao et al., 2016] on caries experience, only few of the included studies reported these data; therefore, their mediating role between dental caries and IBD could not be thoroughly analyzed. Indeed, given the paucity of the included studies, the presence of publication bias could not be completely ruled out. Finally, another concern regards the impossibility to infer neither causality nor temporality between caries and IBD due to the lack of longitudinal studies.

\section{Conclusion}

Our study reveals a remarkably higher past and present occurrence of dental caries in patients with IBD, either CD or UC, when compared to healthy controls. Therefore, we propose that oral health programs, which should include the early detection of dental caries and behavioral interventions for risk factors modification (mainly sugar consumption), be encompassed in the treatment plan of IBD patients from the moment of diagnosis. Further studies are needed to evaluate the presence of a cause-effect relationship between dental caries and IBD.

\section{Statement of Ethics}

An ethics statement is not applicable because this study is based exclusively on published literature.

\section{Conflict of Interest Statement}

The authors have no conflicts of interest to declare. 


\section{Funding Sources}

There are no funding sources related to this study.

\section{Author Contributions}

Crystal Marruganti participated in the conceptual design of the work, data acquisition, statistical analysis, and drafting of the manuscript. Nicola Discepoli participated in the conceptual design of the work and drafting of the manuscript. Carlo Gaeta participated in data acquisition and revision of the manuscript. Giovanni Fran- ciosi participated in data acquisition and revision of the manuscript. Marco Ferrari participated in the revision of the manuscript. Simone Grandini participated in the conceptual design of the work and revision of the manuscript.

\section{Data Availability Statement}

All data generated or analyzed during this study are included in this article, its supplementary material files and the article included in the review. Further enquiries can be directed to the corresponding author.

\section{References}

Abraham C, Cho JH. Inflammatory bowel disease. N Engl J Med. 2009;361(21):2066-78.

Baumgart DC, Sandborn WJ. Crohn's disease. Lancet. 2012;380(9853):1590-605.

Brito F, de Barros FC, Zaltman C, Carvalho AT, Carneiro AJ, Fischer RG, et al. Prevalence of periodontitis and DMFT index in patients with Crohn's disease and ulcerative colitis. J Clin Periodontol. 2008;35(6):55560.

Burisch J, Pedersen N, Cukovic-Cavka S, Turk N, Kaimakliotis I, Duricova D, et al. Environmental factors in a population-based inception cohort of inflammatory bowel disease patients in Europe - an ECCO-EpiCom study. J Crohns Colitis. 2014;8(7):607-16.

Chapman-Kiddell CA, Davies PS, Gillen L, Radford-Smith GL. Role of diet in the development of inflammatory bowel disease. Inflamm Bowel Dis. 2010;16(1):137-51.

DerSimonian R, Laird N. Meta-analysis in clinical trials. Control Clin Trials. 1986;7(3):177-88.

Dotson JL, Hyams JS, Markowitz J, LeLeiko NS, Mack DR, Evans JS, et al. Extraintestinal manifestations of pediatric inflammatory bowel disease and their relation to disease type and severity. J Pediatr Gastroenterol Nutr. 2010; 51(2):140-5.

Gao X, Jiang S, Koh D, Hsu CY. Salivary biomarkers for dental caries. Periodontol 2000. 2016; 70(1):128-41.

Głąbska D, Guzek D, Lech G. Analysis of the nutrients and food products intake of polish males with ulcerative colitis in remission. $\mathrm{Nu}-$ trients. 2019;11(10):1-15.

Grössner-Schreiber B, Fetter T, Hedderich J, Kocher T, Schreiber S, Jepsen S. Prevalence of dental caries and periodontal disease in patients with inflammatory bowel disease: a case-control study. J Clin Periodontol. 2006; 33(7):478-84

Guyatt GH, Oxman AD, Kunz R, Falck-Ytter Y, Vist GE, Liberati A, et al. GRADE: going from evidence to recommendations. Chin J EvidBased Med. 2009a;9(3), 257-9.

Guyatt GH, Oxman AD, Kunz R, Vist GE, FalckYtter Y, Schünemann HJ. GRADE: what is "quality of evidence" and why is it important to clinicians? Chin J Evid-Based Med. 2009b; 9(2):133-7.

Guyatt GH, Oxman AD, Schünemann HJ, Tugwell P, Knottnerus A. GRADE guidelines: a new series of articles in the journal of clinical epidemiology. J Clin Epidemiol. 2011;64(4): $380-2$.

Halme L, Meurman JH, Laine P, von Smitten K, Syrjänen $S$, Lindqvist $C$, et al. Oral findings in patients with active or inactive Crohn's disease. Oral Surg Oral Med Oral Pathol. 1993; 76(2):175-81

Järnerot G, Järnmark I, Nilsson K. Consumption of refined sugar by patients with Crohn's disease, ulcerative colitis, or irritable bowel syndrome. Scand J Gastroenterol. 1983;18(8): 999-1002.

Jørgensen LG, Fredholm L, Hyltoft Petersen P, Hey H, Munkholm P, Brandslund I. How accurate are clinical activity indices for scoring of disease activity in inflammatory bowel disease (IBD)? Clin Chem Lab Med. 2005;43(4): 403-11.

Meurman JH, Halme L, Laine P, von Smitten K, Lindqvist C. Gingival and dental status, salivary acidogenic bacteria, and yeast counts of patients with active or inactive Crohn's disease. Oral Surg Oral Med Oral Pathol. 1994; 77(5):465-8.

Nishida A, Inoue R, Inatomi O, Bamba S, Naito $\mathrm{Y}$, Andoh A. Gut microbiota in the pathogenesis of inflammatory bowel disease. Clin J Gastroenterol. 2018;11(1):1-10.

Ooi CJ, Makharia GK, Hilmi I, Gibson PR, Fock KM, Ahuja V, et al. Asia Pacific consensus statements on Crohn's disease. Part 1: definition, diagnosis, and epidemiology: (Asia Pacific Crohn's disease consensus-part 1). J Gastroenterology Hepatology. 2016;31(1): 45-55.

Ordás I, Eckmann L, Talamini M, Baumgart DC, Sandborn WJ. Ulcerative colitis. Lancet. 2012; 380(9853):1606-19.

Page MJ, McKenzie JE, Bossuyt PM, Boutron I, Hoffmann TC, Mulrow CD, et al. The PRISMA 2020 statement: an updated guideline for reporting systematic reviews. BMJ. 2021;372: n71.
Rodrigues E, Laranjeira N, Nunes G, Roque-Ramos L, Vieira A, Fonseca J. Are cariogenic bacteria the major risk factor to dental caries in patients with ulcerative colitis? Arq Gastroenterol. 2019;56(2):118-23.

Rooney TP. Dental caries prevalence in patients with Crohn's disease. Oral Surg Oral Med Oral Pathol. 1984;57(6):623-4.

Schütz T, Drude C, Paulisch E, Lange KP, Lochs H. Sugar intake, taste changes and dental health in Crohn's disease. Dig Dis. 2003; 21(3):252-7.

Selwitz RH, Ismail AI, Pitts NB. Dental caries. Lancet. 2007;369(9555):51-9.

She YY, Kong XB, Ge YP, Liu ZY, Chen JY, Jiang JW, et al. Periodontitis and inflammatory bowel disease: a meta-analysis. BMC Oral Health. 2020;20(1):67-11.

Sheiham A, James WP. Diet and dental caries: the pivotal role of free sugars reenmphasized. J Dent Res. 2015;94(10):1341-7.

Sheiham A, Watt RG. The common risk factor approach: a rational basis for promoting oral health. Community Dent Oral Epidemiol. 2000;28(6):399-406.

Shivashankar R, Lichtenstein GR. Mimics of inflammatory bowel disease. Inflamm Bowel Dis. 2018;24(11):2315-21.

Ślebioda Z, Szponar E, Linke K. Comparative analysis of the oral cavity status in patients with Crohn's disease and ulcerative colitis. J Stomatol. 2011;64(3-4)382-93.

Stang A. Critical evaluation of the Newcastle-Ottawa scale for the assessment of the quality of nonrandomized studies in meta-analyses. Eur J Epidemiol. 2010;25(9):603-5.

Sundh B, Emilson CG. Salivary and microbial conditions and dental health in patients with Crohn's disease: a 3-year study. Oral Surg Oral Med Oral Pathol. 1989;67(3):286-90.

Szczeklik K, Danuta O, Maciej Ż, JolantaPP, TomaszM. Patients with Crohn's disease living in urban and rural regions of Southern Poland. Med Int Rev. 2017;4(109):280-7.

Szymanska S, Lördal M, Rathnayake N, Gustafsson A, Johannsen A. Dental caries, prevalence and risk factors in patients with Crohn's disease. PLoS One. 2014;9(3):e91059-5. 
Vavricka SR, Manser CN, Hediger S, Vögelin M, Scharl M, Biedermann L, et al. Periodontitis and gingivitis in inflammatory bowel disease: a case-control study. Inflamm Bowel Dis. 2013;19(13):2768-77.

Vavricka SR, Schoepfer A, Scharl M, Lakatos PL, Navarini A, Rogler G. Extraintestinal manifestations of inflammatory bowel disease. Inflamm Bowel Dis. 2015;21(8):1982-92.
Wells GA, Shea B, O'Connel D, Robertson J, Peterson J, Welch V, et al. The Newcastle-Ottawa scale (NOS) for assessing the quailty of nonrandomised studies in meta-analyses. Clin Epidemiol. 2009. Epub ahead of print. http: //www.ohri.ca/programs/clinicalepidemiology/oxford.htm.
World Health Organization. In oral health surveys - basic methods. 5th ed. World Health Organization; 2013. https://www.who.int/ oral_health/publications/9789241548649/ en/.

Zhang L, Gao X, Zhou J, Chen S, Zhang J, Zhang $\mathrm{Y}$, et al. Increased risks of dental caries and periodontal disease in Chinese patients with inflammatory bowel disease. Int Dent J. 2020; 70(3):227-36. 